\title{
Jet-gas interactions at crucial jet power for feedback
}

\author{
D. M. Worrall and M. Birkinshaw \\ HH Wills Physics Laboratory, University of Bristol, Tyndall Avenue, Bristol, BS8 1TL, UK \\ email: d.worrall@bristol.ac.uk
}

\begin{abstract}
Most X-ray studies of radio-mode feedback have concentrated on locally-abundant low-power radio sources in relatively rich cluster environments. But the scaling found between mechanical and radiative power, when combined with the radio luminosity function, means that half of the heating in the local Universe is expected from higher-power sources, which lie within a factor of about three of the FR I/II transition, and these sources encounter a wide range of atmosphere properties. We summarize what is observed at FR I/II transition powers from a complete sample observed with modest Chandra exposure times. We then discuss two systems with deep Chandra data. In one we find that the work done in driving shocks exceeds that in evacuating cavities. This source also displays a remarkable jet-cloud interaction, and revealing hotspot X-ray emission. In the second we find evidence of radio-emitting plasma running along boundaries between gas of different temperature, apparently lubricating the gas flows and inhibiting heat transfer, and itself being heavily structured by the process.
\end{abstract}

Keywords. galaxies: active, galaxies: jets, radio continuum: galaxies, X-rays: galaxies: clusters

\section{Crucial jet power}

A notable achievement of the current generation of X-ray observatories, in particular Chandra, has been the common detection and separation of emission from the jets, knots, hotspots, and lobes of radio galaxies and quasars, leading to greater understanding of source energetics and pressures, and the consequent impact of radio-emitting plasma on the X-ray-emitting environments in which they reside. This has been achieved through observing programs targeting individual sources and moderate-sized samples (see Harris \& Krawczynski (2006) and Worrall (2009) for reviews).

Complementary work has targeted the densest cluster atmospheres, in the context of understanding radio sources as providing the heating required to prevent central gas from cooling below about $1 \mathrm{keV}$ (see McNamara \& Nulsen (2007) and Fabian (2012) for reviews). The Einstein and ROSAT missions had already found evidence that the radio lobes of NGC 1275 has pushed aside X-ray gas in the Perseus cluster (e.g., Böhringer et al. 1993), and with Chandra clusters and groups have been found commonly to harbour gas cavities containing radio plasma that originates from active galaxies (e.g., Bîrzan et al. 2004), and that contain enough enthalpy to balance current radiative cooling (e.g., Dunn et al. 2005, Rafferty et al. 2006, Panagoulia et al. 2014).

While most cavity sources are of reasonably low jet power, correlations between jet and cavity power are found, such as that of Cavagnolo et al. (2010) which stretches over more than six decades (Fig. 1). To find where cavity power is dominant, the radio sources must then be weighted by their space density (Fig. 2). Using the radio luminosity function of Best et al. (2005), we find that one half of the density-weighted cavity power should arise from radio sources with power-densities measured at $1.4 \mathrm{GHz}$ that are within a factor of three of $3 \times 10^{25} \mathrm{~W} \mathrm{~Hz}^{-1}$, i.e., sources in the broad transition zone between FR I and FR II morphologies (Fanaroff \& Riley 1974). We will refer to these as FR I/II transition sources, 


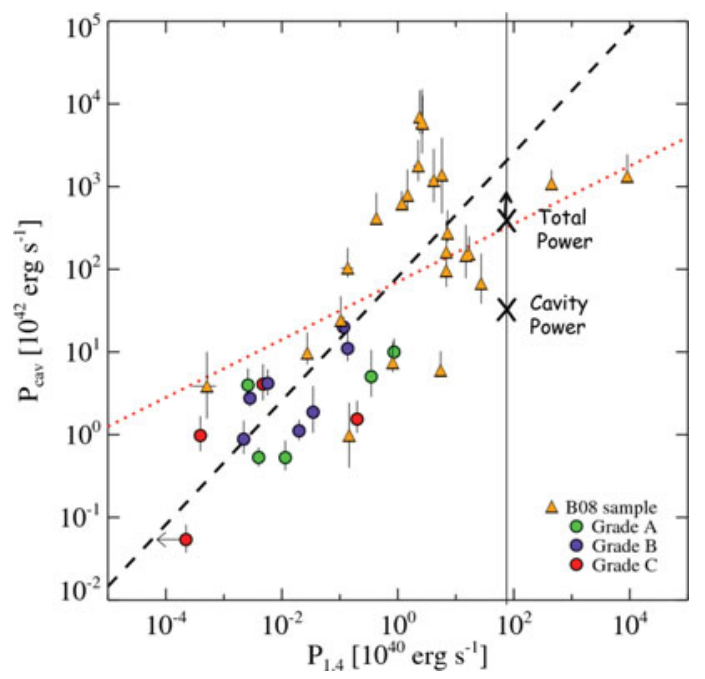

Figure 1. X-ray cavity power against radio power. Plot is adapted from Cavagnolo et al. (2010) to show also the cavity power of PKS B2152-699 (cross), and the lower limit to PKS B2152-699's total power (cross and arrow) after including the kinetic and thermal energy of shocked gas. In the latter case, PKS B2152-699 fits much better the correlation (dashed line).

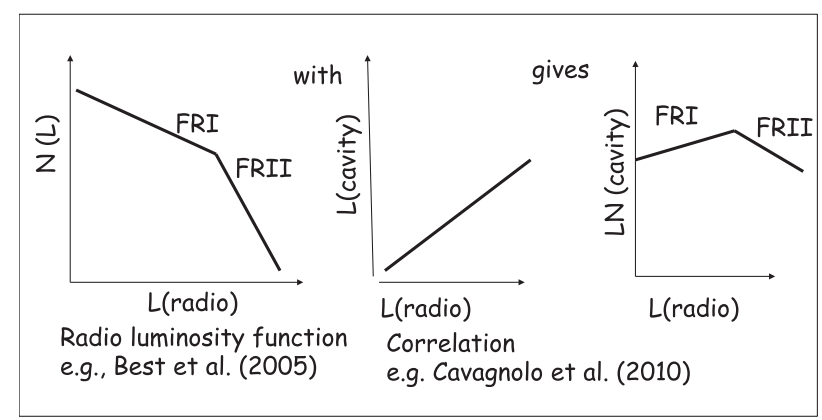

Figure 2. Sketch to illustrate that when the correlation of Fig. 1 (center panel) is weighted by the number density of sources (left panel), the cavity power (or luminosity, $L$ ) in the local Universe is dominated by sources of intermediate radio power (within the FR I/FR II transition zone).

and they are the objects of key importance for targeted study with X-ray observatories if we are to understand 'feedback' selected by the radio-source protagonists rather than selected for the richness or cooling time of their environments.

\section{A complete sample}

There are $16 \mathrm{FRI}$ /II transition sources in the 3CRR catalogue, which contains the brightest sources at $178 \mathrm{MHz}$ in a region of the northern sky (Laing et al. (1983)). Of these 15 are at $z<0.1$ and all have good Chandra observations with a median exposure time of about $43 \mathrm{ks}$. Since they are selected from a complete radio catalogue, they are unbiased with respect to the X-ray-emitting atmosphere. There are a number of papers on their Chandra data, the most relevant for gas interactions and heating being Sun et al. (2005), Hardcastle et al. (2005), Hardcastle et al. (2007), Hodges-Kluck 


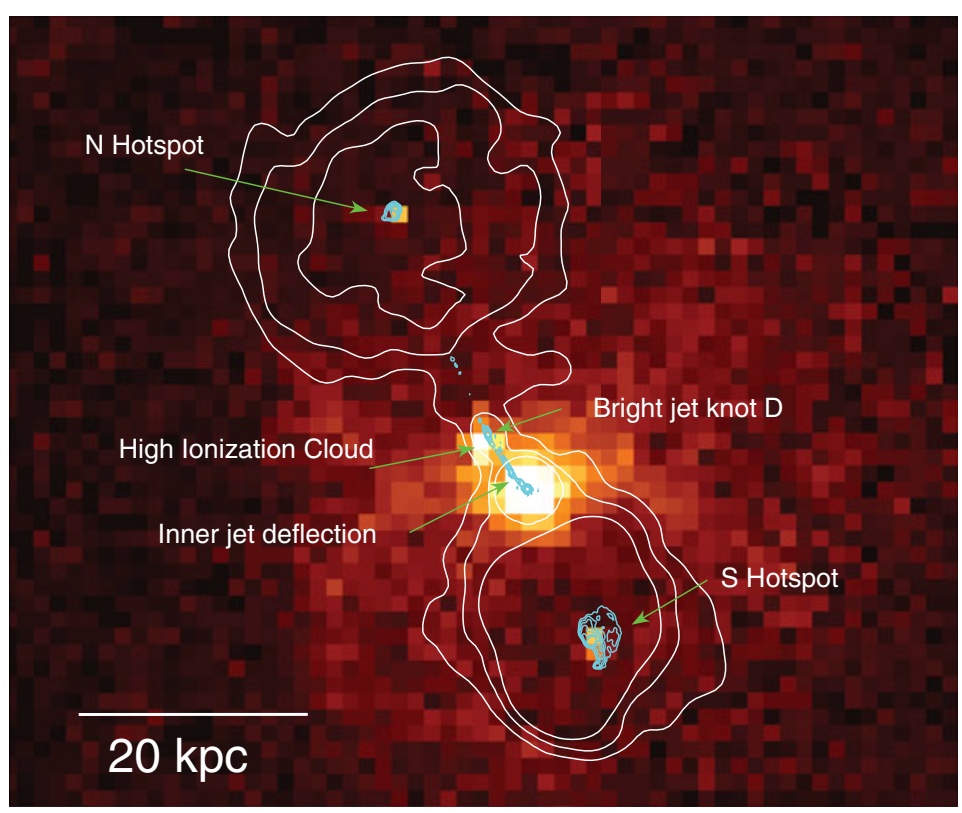

Figure 3. PKS B2152-699 based on Worrall et al. (2012), with high-resolution 15-GHz and low-resolution 5-GHz ATCA radio contours on Chandra data. The radio jet weaves its way from the core through an inner deflection point and bright knot $\mathrm{D}$ which lies next to a high ionization cloud (HIC) which is seen also in its X-ray-emitting gas. The hotpots are prominent in radio and X-ray (Figs. 4 and 5). The lobes occupy X-ray cavities and are embraced by striking density enhancements formed by shocks with Mach number $\approx 2.7$.

et al. (2010), Kraft et al. (2012), Hardcastle et al. (2012), Mannering et al. (2013) and Mannering (2013).

The environments and level of interaction between the radio plasma and gas vary. There are two giants which no longer have a rich atmosphere to heat (DA 240 and 3C 326), and several trail sources shaped by rich atmospheres and ram pressure, or with localized gas interactions (3C 83.1B, 3C 293, 3C 465 and PKS 1227+119). 'Belted' sources are relatively common (Mannering 2013), where radio plasma is driven by gas arising from mergers or fossil groups, and where there is probably little current heating of large-scale X-ray gas, but ongoing local interactions (3C 98, 3C 35, 3C 285, 4C 73.08, 3C 192). Strong shocks are inferred for two sources: a Mach $\sim 2$ shock driven into $k T \approx 0.4$-keV gas by the compact source 3C 305 (Hardcastle et al. 2012), and a Mach $\sim 1.7$ shock driven into $k T \approx 2$ $\mathrm{keV}$ gas by the large (cavity) source 3C 310 (Kraft et al. 2012). Two sources (3C 382 and 3C 390.3) are broad-line radio galaxies with beamed core emission that makes any shocked gas difficult to detect.

The small-number statistics from 3CRR suggest there is less than a 20 per cent chance that a given source is widely heating its interstellar or intracluster medium, but local heating is rather common. More sensitive observations are needed to explore this further, and we turn now to two example fields of FR I/II transition sources (not from 3CRR) where deeper Chandra data are already available.

\section{PKS B2152-699}

PKS B2152-699 at $z=0.0282$ is found to be in a $k T \approx 1 \mathrm{keV}$ group atmosphere (Worrall et al. 2012, Fig. 3). The lobes evacuate clear cavities, and the detection of 


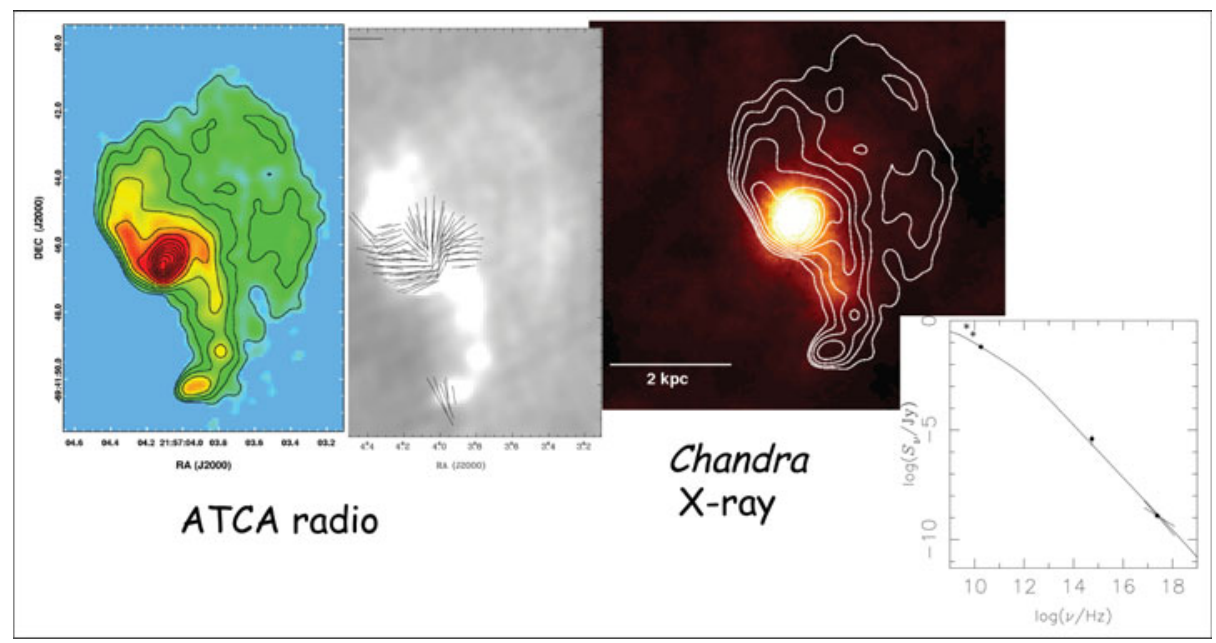

Figure 4. X-ray emission from the southern hotspot matches well the brightest radio regions, and together with ground-based optical data the spectrum is consistent with synchrotron radiation. The $15-\mathrm{GHz}$ fractional polarization $\mathrm{E}$ vectors show a fascinating spiraling magnetic field.

inverse Compton X-ray emission means that the internal lobe energetics can be estimated without needing to rely on an assumption of minimum energy. Density and temperature enhancements of gas around the lobes point to strong shocks, with one of the highest measured Mach numbers of $\sim 2.7$. The strength of the shocks mean that the cavity power, normally used as a proxy for jet power, is dominated by the kinetic and thermal energy of the shocked gas (only a lower limit can be placed on the latter because full heating effects are confused by unshocked gas along the line of sight). Increasing the cavity power to account for the dominant components of jet power leads to the source lying better on the earlier correlations (Fig. 1).

PKS B2152-699 is also remarkable for displaying one of the clearest examples of a jet interacting with a high ionization cloud (HIC), whose location next to a bright jet knot is marked in Figure 3. Velocity structure found in recent integral-field-unit data confirms the interaction and adds substance to estimates of the energetics (Smith et al. 2014).

The hotspot emission is also of particular note. The southern hotpot shows remarkable radio-polarization structure, although the X-ray emission is more normal for a source of its power, with its position matching well the brightest radio region, and being spectrally consistent with synchrotron radiation from the high-energy end of a single electron population (Fig. 4).

In contrast, for the northern hotspot, on the side the jet is observed (also in X-rays out to knot D), the X-rays are distinctly closer to the core than the radio (Fig. 5). We are using new Chandra and HST data to test the idea that this is a result of inverse Compton scattering by a jet entering the hotspot with relatively high bulk Lorentz factor and small angle to the line of sight.

\section{NGC 7016/7018}

The non cool-core cluster A3744 at $z=0.038$ hosts three prominent galaxies, two of which are FR I/II transition sources (Worrall \& Birkinshaw 2014, Fig. 6). A prominent $\mathrm{X}$-ray cavity is located in projection where the radio plasma from the two galaxies most overlaps. The gas in A3744 is too hot by $k T \approx 1.5 \mathrm{keV}$ for its X-ray luminosity compared 


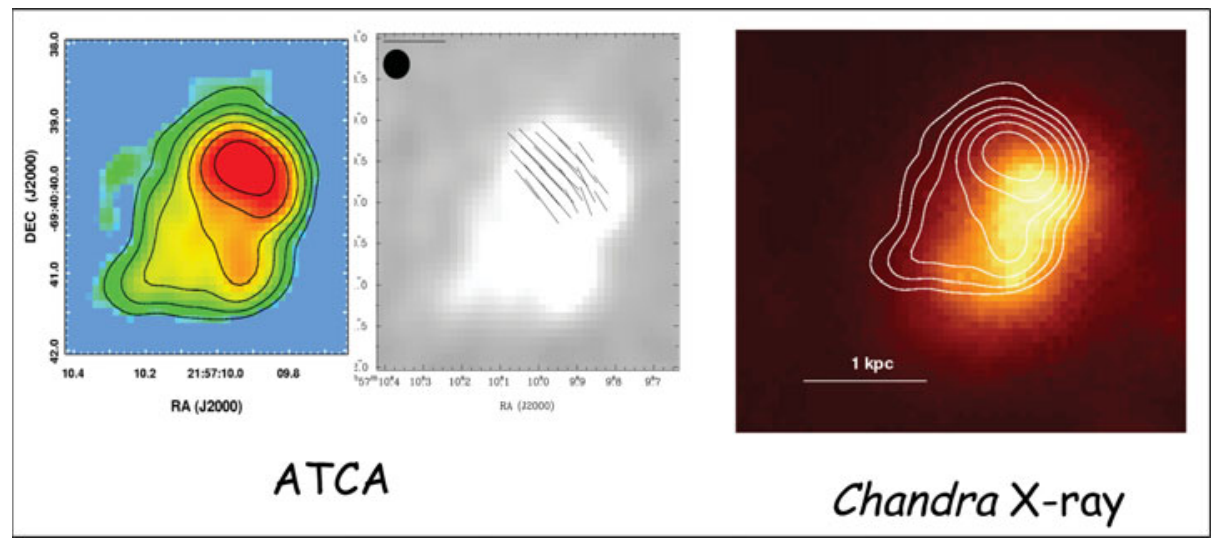

Figure 5. The northern hotspot is less remarkable than the southern one in the radio. But in the X-ray there is a pronounced offset, with X-rays peaking closer to the core than in the radio.
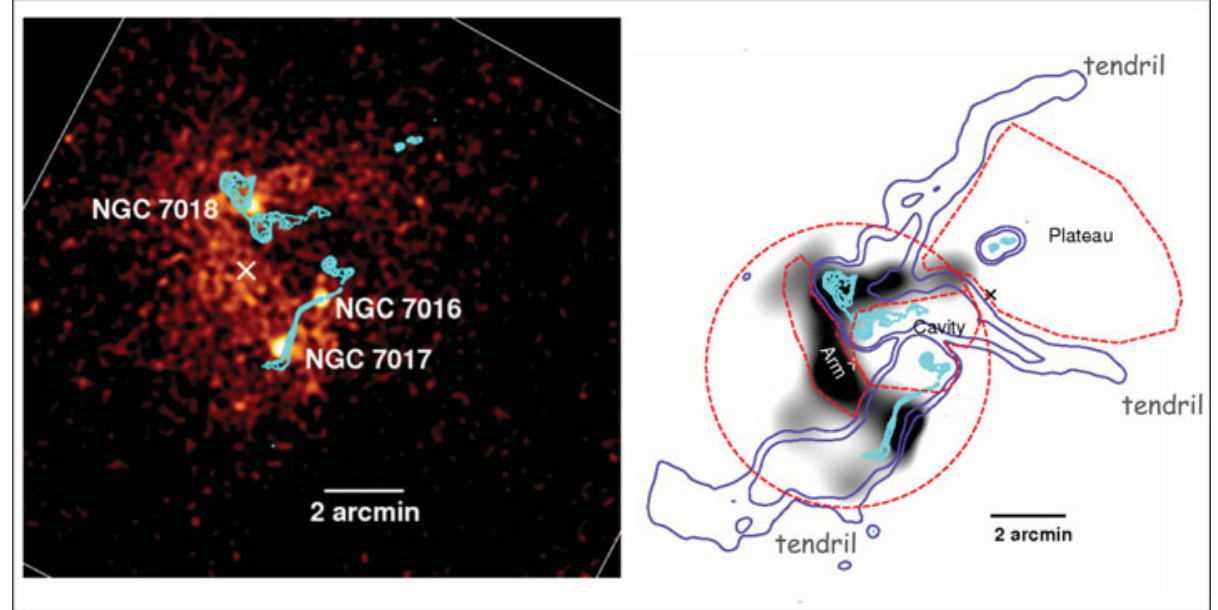

Figure 6. The galaxies and X-ray gas in cluster A3744 based on Worrall \& Birkinshaw 2014. The left panel shows the higher-resolution 1.4-GHz radio contours for NGC 7016 and 7018 on a Chandra image showing emission from three galaxy atmospheres and structured cluster gas. The right panel shows lower-resolution $1.4-\mathrm{GHz}$ contours. The X-ray cavity is co-located in projection with the region of overlapping low-resolution radio emission from the two galaxies. $\mathrm{X}$-ray gas in the plateau region, embraced by low-surface-brightness radio 'tendrils', is cooler than that the other side of the tendrils.

with other clusters, but the single cavity provides too little enthalpy by a factor of about 85 to be the cause, and a recent merger is more likely. This appears to have led to a temperature and density structure in the gas in which the radio tendrils of NGC 7018 hug cooler gas in a 'plateau' region, with hotter gas beyond. There may be similar thermal protection from the tendril of NGC 7016. We suggest that the reason for this is that the magnetized relativistic plasma acts as a barrier to transport (the smaller gyro radius in radio plasma combined with a likely ordered magnetic field would reduce thermal conductivity across the boundary). The radio plasma appears to provide lubrication between the gas layers, helping to preserve post-merger flows. 


\section{Conclusions}

Half of the radio-mode heating in the local Universe should arise from radio sources within 0.3 - 3 of the FR I/II transition power, making these objects of particular importance for deep X-ray and radio study. Unlike typical cavity sources with deep observations, they are not confined to rich clusters. A cursory study of a complete sample of FR I/II transition sources from the 3CRR catalogue suggests a chance of only about $20 \%$ that a source is interacting with or heating large-scale gas now, although local heating appears to be common.

Deep Chanda observations of FR I/II transition sources are yielding interesting results that are breaking some prejudices and pointing to the first observation of some interesting phenomena. For example, in PKS B2152-699 the shocks around the radio lobes are strong, with Mach numbers about 2.7, whereas prevailing wisdom had been in favour of shocks being weak at best (e.g., McNamara \& Nulsen 2007). While cavities are clearly seen, cavity power is dominated by the kinetic and thermal energy of shocked gas, and this extra power is needed to bring the source into reasonable agreement with correlations with jet power (Worrall et al. 2012). The cavity produced by a pair of FR I/II transition sources in the cluster A3744 provides less than two per cent of the excess enthalpy needed to explain the cluster's excess temperature for its luminosity, but that excess is most likely explained by merger activity. Perhaps more importantly, the system provides the first evidence that low-surface-brightness tendrils of radio plasma appear to separate regions of different X-ray gas temperature, apparently lubricating gas flows and inhibiting heat transfer (Worrall \& Birkinshaw 2014). Extrapolating from PKS B2152-699 and A3744, our results suggest that there is much more to be learned from deep Chandra observations of the FRI/II transition population.

\section{References}

Best, P. N., Kauffmann, G., Heckman, T. M., \& Ivezić, Z., 2005, MNRAS, 362, 9

Bîrzan, L., Rafferty, D. A., McNamara, B. R., Wise, M. W., \& Nulsen, P. E. J., 2004, ApJ, 607, 800

Böhringer, H., Voges, W., Fabian, A. C., Edge, A. C., \& Neumann, D. M., 1993, MNRAS, 264, L25

Cavagnolo, K. W., McNamara, B. R., Nulsen, P. E. J., et al., 2010, ApJ, 720, 1066

Dunn, R. J. H., Fabian, A. C., \& Taylor, G. B., 2005, MNRAS, 364, 1343

Fabian, A. C., 2012, ARAA, 50, 455

Fanaroff, B. L. \& Riley, J. M., 1974, MNRAS, 167, 31P

Hardcastle, M. J., Sakelliou, I., \& Worrall, D. M., 2005, MNRAS, 359, 1007

Hardcastle, M. J., Kraft, R. P., Worrall, D. M., et al., 2007, ApJ, 662, 166

Hardcastle, M. J. et al. 2012, MNRAS, 424, 1774

Harris, D. E. \& Krawcynski, H., 2006, ARAA, 44, 463

Hodges-Kluck, E. J., Reynolds, C. S., Cheung, C. C., \& Miller, M. C., 2010, ApJ, 710, 1205

Kraft, R. P., et al. 2012, ApJ, 749, 19

Laing, R. A., Riley, J. M., \& Longair, M. S. 1983, MNRAS, 204, 151

Mannering, E. J. A., 2013, Ph. D. thesis, University of Bristol

Mannering, E. J. A., Worrall, D. M., \& Birkinshaw, M., 2013, MNRAS, 431, 858

McNamara, B. R. \& Nulsen, P. E. J., 2007, ARAA, 45, 117

Panagoulia, E. K., Fabian, A. C., Sanders, J. S., \& Hlavacek-Larrondo, J., 2014, MNRAS, 444, 1236

Rafferty, D. A., McNamara, B. R., Nulsen, P. E. J., \& Wise, M. W., 2006, ApJ, 652, 216

Smith, D., Young, A. J., Worrall, D. M., \& Birkinshaw, M., 2014, in preparation

Sun, M., Jerius, D., \& Jones, C., 2005, ApJ, 633, 165

Worrall, D. M., 2009, A\&AR, 17, 1

Worrall, D. M. \& Birkinshaw, M., 2014, ApJ, 784, 36

Worrall, D. M., Birkinshaw, M., Young, A. J., et al., 2012, MNRAS, 424, 1346 